\title{
The Effects of Surfaces on the Aerodynamics and Acoustics of Jet Flows
}

\author{
Matthew J. Smith* \\ National Institute of Aerospace, Hampton, Virginia 23681 \\ Steven A. E. Miller ${ }^{\dagger}$ \\ NASA Langley Research Center, Hampton, Virginia 23681
}

\begin{abstract}
Aircraft noise mitigation is an ongoing challenge for the aeronautics research community. In response to this challenge, low-noise aircraft concepts have been developed that exhibit situations where the jet exhaust interacts with an airframe surface. Jet flows interacting with nearby surfaces manifest a complex behavior in which acoustic and aerodynamic characteristics are altered. In this paper, the variation of the aerodynamics, acoustic source, and far-field acoustic intensity are examined as a large flat plate is positioned relative to the nozzle exit. Steady Reynolds-Averaged Navier-Stokes solutions are examined to study the aerodynamic changes in the field-variables and turbulence statistics. The mixing noise model of Tam and Auriault is used to predict the noise produced by the jet. To validate both the aerodynamic and the noise prediction models, results are compared with Particle Image Velocimetry (PIV) and free-field acoustic data respectively. The variation of the aerodynamic quantities and noise source are examined by comparing predictions from various jet and flat plate configurations with an isolated jet. To quantify the propulsion airframe aeroacoustic installation effects on the aerodynamic noise source, a non-dimensional number is formed that contains the flow-conditions and airframe installation parameters.
\end{abstract}

\section{Nomenclature}

$\begin{array}{ll}\text { Abbreviations } \\ C F D & \text { Computational Fluid Dynamics } \\ F U N 3 D & \text { Fully Unstructured Navier-Stokes Three-Dimensional Solver } \\ H W B & \text { Hybrid Wing Body } \\ N P R & \text { Nozzle Pressure Ratio } \\ O A S P L & \text { Overall Sound Pressure Level } \\ P A A & \text { Propulsion Airframe Aeroacoustics } \\ P I V & \text { Particle Image Velocimetry } \\ P S D & \text { Power Spectral Density } \\ R A N S & \text { Reynolds-Averaged Navier-Stokes } \\ S H J A R & \text { Small Hot Jet Acoustic Rig } \\ S M C & \text { Small Metal Chevron } \\ S P L & \text { Sound Pressure Level } \\ S S T & \text { Shear Stress Transport } \\ T K E & \text { Turbulent Kinetic Energy } \\ T T R & \text { Total Temperature Ratio } \\ \text { Symbols } & \\ A & \text { Empirical constant } \\ c & \text { Speed of sound } \\ c_{\infty} & \text { Free-stream speed of sound }\end{array}$

\footnotetext{
* Graduate Research Assistant, Department of Aerospace and Ocean Engineering, Virginia Tech, AIAA Member.

${ }^{\dagger}$ Research Aerospace Engineer, Aeroacoustics Branch, NASA Langley Research Center, 2 N. Dryden St. MS 461, Hampton, VA, 23681, USA, AIAA Member, s.miller@nasa.gov
} 


\begin{tabular}{|c|c|}
\hline$c_{\tau}$ & Empirical constant associated with turbulent time scale \\
\hline$c_{l}$ & Empirical constant associated with turbulent length scale \\
\hline$D$ & Nozzle exit diameter \\
\hline$D_{j}$ & Fully expanded jet diameter \\
\hline$k_{s}$ & Turbulent kinetic energy associated with fine-scale mixing noise \\
\hline$l_{s}$ & Turbulent length scale \\
\hline$M_{a}$ & Acoustic Mach number \\
\hline$M_{d}$ & Design Mach number \\
\hline$M_{j}$ & Fully expanded Mach number \\
\hline$M_{\infty}$ & Free-stream Mach number \\
\hline$p$ & Static pressure \\
\hline$p_{a}$ & Acoustic pressure \\
\hline$p_{j}$ & Fully expanded static pressure \\
\hline$p_{o}$ & Stagnation pressure \\
\hline$\hat{q}_{s}$ & Statistical source term \\
\hline$S t$ & Strouhal number \\
\hline$S$ & Spectral density of acoustic pressure \\
\hline$T_{0}$ & Stagnation temperature \\
\hline$T_{j}$ & Fully expanded static temperature \\
\hline $\bar{u}$ & Mean streamwise velocity component \\
\hline$u_{j}$ & Fully expanded jet velocity \\
\hline $\boldsymbol{x}=\boldsymbol{x}(x, y, z)$ & Vector observer position \\
\hline$x_{I}$ & Estimate of jet impingement location \\
\hline$x_{p}$ & Axial distance to the plate trailing edge \\
\hline $\boldsymbol{y}=\boldsymbol{y}(x, y, z)$ & Source vector \\
\hline$y_{p}$ & Radial distance from the plate to the jet centerline \\
\hline$\delta_{\eta}$ & Spreading angle of the jet \\
\hline$\epsilon$ & Dissipation of turbulent kinetic energy \\
\hline$\Gamma$ & Non-dimensional number \\
\hline$\gamma$ & Ratio of specific heats \\
\hline$\tau_{s}$ & Turbulent time scale \\
\hline$\omega$ & Radial frequency \\
\hline$\psi$ & Observer angle from the upstream axis \\
\hline
\end{tabular}

\section{Introduction}

Aircraft noise mitigation is an ongoing challenge for the aeronautics research community. Noise associated issues such as acoustic fatigue, annoyance to the public, and hearing loss have led to increased efforts toward the reduction of jet noise. Increased understanding of jet noise produced in various flow conditions allows for improved acoustic prediction models, that are essential for the development of new low-noise aircraft designs. In support of these aircraft designs, shielding techniques have been studied that involve airframe structures located near jet engines to minimize noise perceived by the observer on the ground (see for example Papamoschou and Mayoral $^{1}$ or Huang and Papamoschou ${ }^{2}$ ). Jet flows interacting with these nearby surfaces result in complex scattered acoustic fields that are difficult to model.

Supersonic aircraft configurations, such as those developed by Welge et $a l^{3,4}$ for the 2020 to 2035 timeframe or by Morgenstern et al. ${ }^{5}$ for the 2035 timeframe and beyond, demonstrate complicated nozzle and engine placement designed to reduce sonic boom and jet noise. Advanced low bypass ratio engines, as shown by Sokhey and Kube-McDowell, ${ }^{6}$ can be integrated into the airframe while potentially reducing jet noise. These concepts could also exhibit situations where the jet exhaust interacts directly with an airframe surface resulting in additional noise sources and a complicated scattered field. New jet noise prediction methodologies need to be developed to address these complicated configurations and advanced nozzle designs.

Another advanced concept is the Hybrid Wing Body (HWB) aircraft that shields jet noise by placing the engines above the airframe. This effect is described by Thomas et al. ${ }^{7}$ and Czech et al. ${ }^{8}$ Several techniques 
have been developed to predict the scattered noise from the engine. Some of these techniques include the Boundary Element Method, ${ }^{2}$ the Equivalent Source Method used in NASA's Fast Scattering Code,${ }^{9}$ and the ray tracing method. ${ }^{10}$ However, only a limited number of source models have been developed for use with scattering methodologies.

Jet surface interactions produce a change in the noise sources associated with the aerodynamics of the jet flow. There have been several studies that examined the aerodynamic effects of nearby and impinging surfaces on jet flows. Donaldson and Snedeker ${ }^{11}$ and Lamont and Hunt ${ }^{12}$ showed that jets impinging on oblique and perpendicular surfaces significantly affect the turbulence and shock cell structure of supersonic jets. Studies involving jets not directly impinging on surfaces include those by Sawyer ${ }^{13}$ and Al-Qutub and Budair ${ }^{14}$ among others. Capturing the effect on the acoustic source due to the changes in the aerodynamic characteristics of the jet flow-field is essential for accurate prediction of jet noise. The effect of nearby surfaces on the aerodynamic flow-field is dependent on multiple parameters such as NPR, TTR, nozzle geometry, the boundary condition on the surface, and the position of the surface relative to the jet.

In this paper, the variation of the aerodynamics, acoustic source, and far-field acoustic intensity are examined as a large flat plate is moved relative to the nozzle exit. Steady Reynolds-Averaged Navier-Stokes (RANS) solutions are found using realistic nozzle and flat plate geometries to study the aerodynamic changes in the field-variables and turbulence statistics. The semi-empirical fine-scale mixing noise model of Tam and Auriault ${ }^{15}$ is used to predict the noise produced by the jet. This model is also used to examine the integrand of the acoustic source that uses quantities obtained from the steady RANS solution as its argument. To validate both the aerodynamic and the noise models, results are compared with Particle Image Velocimetry (PIV) and free-field acoustic data, respectively. The variation of the field-variables, turbulence statistics, and noise is examined by comparing predictions from various jet and plate configurations with an isolated jet. Multiple jet operating conditions are also examined. A non-dimensional number is developed to quantify the effect of the surface on the aerodynamic noise source based on the jet operating condition and surface location.

In the following sections of this paper, a non-dimensional number is introduced to quantify the effect of the airframe on the aerodynamic source, and the associated parameters describing the jet condition and surface location are defined. Next, the steady RANS model is presented and compared with the PIV data of Bridges and Wernet. ${ }^{16}$ Solutions of the steady RANS model are shown for multiple jet and plate configurations. Following the steady RANS assessment, a statistical noise prediction approach is described and compared with measurements of Bridges and Brown. ${ }^{17}$ Then, noise predictions of the isolated jet are compared to the jet with a flat plate at various positions. Finally, a conclusion of this study and related future work are discussed.

\section{Analysis}

The method by which a jet engine is integrated with the airframe of a flight vehicle can have significant effect on the aerodynamic source of sound. This is a highly complicated problem that has received significant attention. ${ }^{7,8,18}$ It is characterized by a large number of parameters that are highly interdependent. A nondimensional number is formed, which has arguments involving the flow-conditions and jet position, that can be used as a basic guide to ascertain whether the aerodynamic source is affected by the airframe relative to the equivalent isolated jet aerodynamic source.

The coordinate system and geometry are illustrated in Fig. 1. Coordinates $x, y$, and $z$ are normalized by the nozzle diameter. This normalized coordinate system is used to illustrate results in the following sections. The origin of the coordinate system used for the analysis is located at the nozzle exit. The positive $x$ axis points in the jet principal direction, the $y$ axis is normal to the plate, and the $z$ axis is parallel to the plate. The plate is located at multiple cross-stream distances from the jet centerline axis to the plate surface, $y_{p}$, and multiple streamwise distances from the nozzle exit to the trailing edge, $x_{p}$.

Parameters are identified that have a direct impact on the aerodynamic source of the jet based on the position of the airframe surface. These parameters are illustrated in Fig. 1. The first parameter is the fully expanded diameter of the jet,

$$
D_{j}=D \sqrt{\frac{M_{d}}{M_{j}}}\left(\frac{1+\frac{\gamma-1}{2} M_{j}^{2}}{1+\frac{\gamma-1}{2} M_{d}^{2}}\right)^{(\gamma+1) / 4(\gamma-1)},
$$


where $D$ is the nozzle exit diameter, $D_{j}$ represents the necessary equivalent nozzle exit diameter for a shock free flow, $M_{d}$ and $M_{j}$ represent the design Mach number and the fully expanded Mach number, respectively, and $\gamma$ is the ratio of specific heats.

Additionally, $x_{p}$ is the distance from the nozzle exit to the trailing edge of the airframe ${ }^{\mathrm{a}}$ parallel to the jet centerline. $y_{p}$ is the characteristic length from the nozzle centerline to the nearest airframe surface. $x_{I}$ is the shadow distance on the airframe surface from the nearest airframe surface point at which the jet first interacts with the airframe. This value is dependent on the problem geometry, the engine cycle condition, and $x_{I}$. The simplest estimate for $x_{I}$ is the jet impingement location, that is a function of the initial spreading rate of the jet. Here $x_{I}$ is approximated as,

$$
x_{I}=\frac{y_{p}-D / 2}{\tan \left[\delta_{\eta}\right]},
$$

where $\delta_{\eta}$ is the spreading angle of the jet. Values of $\delta_{\eta}$ are not readily available without numerical calculations or measurement. Here, we adopt an empirical model developed by Lau ${ }^{19}$ for $\delta_{\eta}$,

$$
\delta_{\eta}=0.177\left(1-0.294 M_{j}^{2}\right)\left(1+\frac{1}{2}\left(M_{j}^{2}-1\right)\left(T_{j} / T_{o}-1.4\right)^{2}\right),
$$

which is valid for a wide range of single-stream jet Mach numbers and temperature ratios. The flows of this investigation fall within the range of validity of Eqn. 3. Equation 3 is dependent on a wide range of parameters that do not explicitly appear. Using these parameters a non-dimensional number is proposed,

$$
\Gamma=\left(\frac{D_{j}}{y_{p}}\right)\left(\frac{x_{p}}{x_{I}}\right) .
$$

Alternatively, Eqn. 4 can be written as,

$$
\Gamma=\frac{D_{j} x_{p} \tan \left[\delta_{\eta}\right]}{y_{p}\left(y_{p}-D / 2\right)}
$$

where $y_{p}>D / 2$. Physically, Eqn. 5 is the ratio of the product of the jet and airframe length scales divided by the cross-stream length scale and interaction distance. Small values of $\Gamma$ can suggest that airframe effects on the jet aerodynamic noise sources are negligible. Likewise, large values of $\Gamma$ can suggest that the effects of the airframe on the aerodynamic noise sources of the jet are very large. In the following sections, Eqn. 5 is evaluated using different jet conditions and airframe surface positions, and compared with numerical predictions of the variation of the noise from the aerodynamic source.

\section{Results}

\section{Steady RANS Assessment}

The steady RANS equations are solved with a Computational Fluid Dynamics (CFD) approach using the NASA Fully Unstructured Navier-Stokes three-dimensional ${ }^{20}$ (FUN3D) solver. The Menter ${ }^{21}$ Shear Stress Transport (SST) turbulence model is used to close the RANS equations. The model utilizes the strengths of the Jones and Launder $k-\epsilon$ model $^{22}$ and the Wilcox $k-\omega$ model. ${ }^{23,24}$ It utilizes the $k$ - $\omega$ model in the inner region of the boundary layer and switches to the $k-\epsilon$ model in the outer region and in free shear layers. The model also takes into account the transport of the principal turbulent shear stress within adverse pressure gradients. In this work, the noise source is dependent upon quantities obtained from the steady RANS solution.

The plate positions relative to the nozzle exit assessed in this paper are shown in Table 1. The first column contains normalized streamwise distances from the nozzle exit to the edge of the flat plate and the second column shows normalized radial distances to the flat plate. Table 2 provides information regarding the jet operating conditions analyzed. The operating conditions include three subsonic jets, one of which is heated, and two cold supersonic jets, one of which is over-expanded and the other ideally-expanded.

The field variables and turbulent statistics are discretized on a mixed element (structured-unstructured) computational grid. An unstructured grid is used to resolve the jet flow when the plate is present while a

\footnotetext{
${ }^{a}$ We elect to use airframe and flat plate interchangeably in terms of the analysis.
} 
structured grid is retained to resolve the jet plume. All calculations are fully three-dimensional and utilize symmetry. The nozzle geometries and flow conditions coincide with the PIV experiment of Bridges and Wernet. ${ }^{16}$ Figure 2 shows an outline of the three-dimensional computational domain with the plate located two nozzle diameters, $D$, offset from the jet axis and parallel to the jet centerline. Values of $p_{o}$ and $T_{o}$ are specified by boundary conditions consisting of nozzle pressure and temperature ratios at the nozzle inlet and static pressure at the domain exit. A symmetric boundary condition is enforced on the $x-y$ plane at $z=0$. All other boundaries are defined with a free-stream condition of $M_{\infty}=0.01$ and ambient pressure. The SMC000 nozzle profile and a portion of the computational domain are shown in Fig. 3. This portion of the domain represents a slice in the $x-y$ plane at $z=0$ normalized by $D$. The full computational domain extends $75 \mathrm{D}$ downstream, $50 \mathrm{D}$ cross-stream along the $z$ axis, and $100 \mathrm{D}$ cross-stream along the $y$ axis to form a rectangular prism. The number of grid points in the domain with a plate present is 2,195,937 and the number of elements is $2,827,906$. The number of grid points in the domain without a plate present is $1,180,575$ and the number of elements is $1,472,843$. These represent typical values for the cases examined.

Validation of the isolated jet steady RANS solution is performed by comparing the streamwise velocity component and Turbulent Kinetic Energy (TKE) with the PIV data from the isolated jet experiment of Bridges and Wernet. ${ }^{16}$ The PIV dataset of Bridges and Wernet ${ }^{16}$ was developed in part to support the validation of jet noise prediction methodologies and steady RANS solutions for a wide range of jet velocities and temperatures. Mean velocities, TKE, and Reynolds stresses are important parameters for statistical acoustic analogies. The first two are used for validation and subsequent analysis in this study.

The first comparison of steady RANS solutions with measurement is performed with a subsonic cold jet operating at $M_{j}=0.513$ from the convergent SMC000 nozzle. Contour plots of Fig. 4 show a qualitative comparison of the streamwise velocity component and TKE. The velocity and TKE are normalized by the fully expanded velocity and its square, respectively. The steady RANS solutions are shown on the top half planes and the corresponding PIV data are shown on the bottom half planes. The streamwise velocity shown in Fig. 4(a) has noticeable variation from the PIV. The predicted thickness of the potential core past $6 D$ is below measurement and the fall-off past $8 D$ is higher. The predicted peak TKE occurs near $5.5 D$ and the PIV peak TKE occurs further downstream at $6.25 D$. Furthermore, the predicted magnitude of peak TKE is lower than measurement. However, the global qualitative agreement is favorable compared to other solutions produced by similar CFD codes (for example see Georgiadis et al. ${ }^{25}$ ).

Qualitative comparisons are conducted for the isolated jet by examining aerodynamic data along the centerline and axial locations at $x / D=1,4$, and 16 . Figure 5 shows the centerline variation of the normalized streamwise velocity component and TKE of both the steady RANS solution and PIV data. Figure 6 shows radial profile comparisons of the same quantities. Figure 5(a) shows that the jet potential core length is larger than measurement by $2 D$. The predicted streamwise velocity component follows the same $\approx 1 / x$ decay as the measured data along the centerline, shown in Fig. 5(a), and also matches the radial decay at $x / D=1$ and $x / D=4$, shown in Fig. 6 (a). The predicted TKE along the centerline matches the experiment in terms of peak magnitude but the peak occurs one diameter further upstream than experiment. The peak magnitudes of TKE at $x / D=1$ and $x / D=4$ are larger than measurement by 0.0015 and $0.001 T K E / U_{j}{ }^{2}$ respectively and are located $0.25 \mathrm{D}$ closer to the centerline than experiment. The solution under-predicts both the streamwise velocity component and TKE relative to measurement far downstream from the nozzle exit.

Qualitative comparisons are also conducted for the supersonic over-expanded jet operating at $M_{j}=1.29$ from the $M_{d}=1.5 \mathrm{SMC016}$ nozzle. Again, the aerodynamic data is examined along the centerline and radial locations at $x / D=1,4$, and 16 . Figure 7 shows the centerline variation of the normalized streamwise velocity component and TKE of both the steady RANS solution and PIV data. Figure 8 shows radial profile comparisons of the same quantities. As seen in Fig. 7, the predicted potential core length is $2 D$ larger relative to measurement. Within the potential core, the predicted shock cell structure locations compare favorably with the experiment. The steady RANS solution over-predicts the rate of decay along the centerline shown in Fig. 7(a) and matches the radial decay in the potential core region at $x / D=1$ and 4 as shown in Fig. 8(a). The steady RANS solution under-predicts the radial decay through the shear layer greater than $0.5 D$ from the centerline. In the fully developed region of the flow at $x / D=16$, the steady RANS solution only slightly over-predicts the radial velocity profile. This is a favorable prediction relative to the TKE profile at $x / D=16$ shown in Fig. 8(b) and the subsonic jet predictions shown at $x / D=16$ in Fig. 6 . On the centerline the peak $T K E / U_{j}{ }^{2}$ is 0.03 below measurement and occurs $2 D$ further downstream than measurement as shown in Fig. $7(\mathrm{~b})$. The TKE predictions match the experiment in peak magnitude at $x / D=4$ but over-predicts 
$T K E / U_{j}^{2}$ at $x / D=1$ and $x / D=16$ by 0.015 and 0.005 , respectively. In concurrence with the subsonic cold jet comparisons of Fig. 6(b), the predicted TKE is lower than measurement further downstream from the nozzle. These trends are representative of all jet conditions shown in Table 2. The predictions are favorable relative to experiment, for all jets examined, in the potential core region of the flow-field where inviscid terms dominate the equations of motion.

The steady RANS solutions of a $M_{j}=0.513$ and $T T R=1.00$ jet from the convergent SMC000 nozzle in the presence of a flat plate are now compared with the isolated jet operating at the same conditions. Figure 9 shows radial profiles of TKE at $x / D=10$ for multiple plate positions. The plate is located at $y_{p} / D=-1,-2,-4,-6,-8$, and -10 perpendicular to the jet centerline and extends to $x_{p} / D=10$ and 20 downstream from the nozzle exit. Figure 9 shows that as the plate is moved closer to the jet the TKE distribution is increasingly deformed. The peak magnitude closest to the plate decreases and the peak magnitude furthest from the plate increases. This trend is amplified when increasing the $x_{p} / D$ location from 10 to 20 for most of the cases examined. Furthermore, the jet plume is being deformed and is drawn toward the plate due to a coandă like effect. For example, in Fig. 9(a) the $x_{p} / D=10$ and isolated jet case both have a TKE local minimum at $y / D=0$. However, as the plate is moved closer as in Fig. 9(f) the local minimum of TKE for $x_{p} / D=10$ is now located at $y / D=-0.16$. The effect of extending the plate from $x_{p} / D=10$ to $x_{p} / D=20$ amplifies the effect of the deformation of the jet plume as can be seen in Fig. 9(a). The peak TKE close to the plate is lower by $0.0005 T K E / U_{j}{ }^{2}$, and the peak TKE furthest from the plate is higher by $0.001 T K E / U_{j}{ }^{2}$. Similar changes of the TKE distribution are observed with all jet conditions examined. These preliminary numerical results show the changes in the aerodynamic characteristics of the jet plume induced by nearby surfaces. These changes effect the noise source amplitude and position as the plate location is altered.

\section{Aeroacoustic Assessment}

The results of the aerodynamic assessment show that nearby solid surfaces change the aerodynamic characteristics of the jet flow-field, even if the jet centerline is multiple diameters away from a solid surface. To assess the changes of the acoustic source quantitatively, a statistical noise prediction approach is selected. Here, we choose the semi-empirical fine-scale mixing noise model of Tam and Auriault. ${ }^{15}$ The expression for the spectral density of the acoustic pressure in the far-field, $S$, is given by,

$$
S(\boldsymbol{x}, \omega)=4 \pi\left(\frac{\pi}{\ln 2}\right)^{3 / 2} \int_{-\infty}^{\infty} \int_{-\infty}^{\infty} \int_{-\infty}^{\infty} \frac{\hat{q}_{s}^{2} l_{s}^{3}}{\bar{c}^{2} \tau_{s}} \frac{\left|p_{a}(\boldsymbol{x} ; \boldsymbol{y}, \omega)\right|^{2} \exp \left[-\frac{\omega^{2} l_{s}^{2}}{\bar{u}^{2} 4 \ln 2}\right]}{1+\omega^{2} \tau_{s}^{2}\left(1-\frac{\bar{u}}{c_{\infty}} \cos \theta\right)^{2}} d \boldsymbol{y}
$$

where $A$ is a constant coefficient, $c$ is the speed of sound, $\bar{u}$ is the mean streamwise velocity component, $\boldsymbol{x}$ is the observer position, $\boldsymbol{y}$ is the source position, and $\omega$ is the radial frequency. $\hat{q}_{s}=(4 / 9) A^{2} \bar{c}^{2} \bar{\rho}^{2} k_{s}^{2}$ is a statistical source term where $k_{s}$ is the TKE associated with turbulence that produces fine-scale mixing noise. $k_{s}$ is set equal to the TKE computed from the steady RANS solution.

If predictions are restricted to the sideline direction and it is assumed that sound refraction by the jet shear layer has negligible effect on $S$, then a simplified form of $p_{a}(\boldsymbol{x} ; \boldsymbol{y}, \omega)$ can be constructed. As shown by Morris and Farassat ${ }^{26}$ the adjoint acoustic pressure at $\psi=\pi / 2$ (the jet sideline) is,

$$
\left|p_{a}\left(x_{2} ; \boldsymbol{y}, \omega\right)\right|^{2}=\frac{\omega^{2}}{64 \pi^{4} c_{\infty}^{4} x^{2}}
$$

This approach does not take into account propagation effects associated with sound waves interacting with surfaces such as the flat plate. This is advantageous because the effects of surfaces on the jet noise source are isolated.

The scales of turbulence in Eqn. 6 are related to the steady RANS solution of the jet by simple dimensional models. The length scale is $l_{s}=c_{l} k_{s}^{3 / 2} / \epsilon$ and the time scale is $\tau_{s}=c_{\tau} k_{s} / \epsilon$, where $\epsilon$ is the dissipation of TKE. The quantity, $\epsilon$, is computed from the steady RANS solution as $\epsilon=0.09 k_{s} \omega$. By relating the turbulent scales to the steady RANS solution, the predicted noise is dependent on the jet mean flow. The mean flow is dependent on the boundary conditions, the nozzle geometry, and the position of the plate relative to the jet. The acoustic source strength and spatial distribution as a function of frequency is directly connected to the steady RANS solutions. 
The coefficients $A, c_{l}$, and $c_{\tau}$ of Eqn. 6 are specified by comparing prediction with measurement, following the methodology of Tam and Auriault. ${ }^{15}$ The coefficients are calibrated with the SMC000 nozzle operating at $M_{j}=1.00$ and $T T R=1.00$. The coefficients are constant irrespective of observer position, jet operating condition, or airframe geometry after calibration. The values are $A=3548.0, c_{l}=0.018$, and $c_{\tau}=0.015$, that vary from those calculated by Tam and Auriault ${ }^{15}$ because the steady RANS solver and turbulence model differ. Figure 10 shows the prediction compared with the experiment of Bridges and Brown. ${ }^{17}$ The Sound Pressure Level (SPL) per unit $S t$ is represented on the $y$-axis, where $S t=\omega D_{j} / u_{j}$ is the Strouhal number. The prediction captures the peak intensity and the nearly correct decay of intensity at high frequency. There is slight discrepancy at lower frequencies where the decay of intensity is lower than measurement.

The effect that the flat plate has on acoustic intensity originating from the jet aerodynamic source is investigated by using the steady RANS solutions and Eqn. 6 . The isolated jet predictions are subtracted from the installed jet predictions on a Power Spectral Density (PSD) basis for comparison. The first comparison consists of the cold subsonic jet at $M_{j}=0.513$ and is presented in Fig. 11. In Fig. 11(a), the plate is located at $y_{p} / D=-1,-2,-4,-6,-8$, and -10 from the jet centerline and extends $x_{p} / D=10$ downstream of the nozzle exit. In Fig. 11(b), the plate is located at $y_{p} / D=-1$ from the jet centerline and extends $x_{p} / D=4,10$, and 20 downstream of the nozzle exit. When the plate is extended to $x_{p} / D=10$ downstream of the nozzle exit, the results from Fig. 11(a) show only a small effect on the noise spectrum as the plate approaches the centerline until the plate is at $y_{p} / D=-2$. The noise deviation from the isolated jet reaches a maximum of $-1.5 d B$ at the lowest frequency and $0.75 d B$ at the highest frequency when the plate is located at $y_{p} / D=-2$. For all plate locations further than $2 D$ away from the centerline, the noise deviations are within $\pm 0.75 \mathrm{~dB}$ from the isolated jet case. When the plate is located at $y_{p} / D=-1$, the noise intensity is $-6.9 d B$ relative to the isolated jet case at the lowest frequency. The effect of varying the plate length relative to the nozzle exit is displayed in Fig. 11(b). At the lowest frequency, the noise intensity deviation from the isolated jet reaches a maximum of $-6.5 d B,-6.9 d B$, and $-7.9 d B$ as the plate is extended to $x_{p} / D=4,10$, and 20 downstream from the nozzle exit, respectively. At the highest frequency, the maximum deviation for each plate extension does not exceed $0.75 \mathrm{~dB}$.

Predictions of the over-expanded supersonic $M_{j}=1.29$ jet from the convergent-divergent SMC016 nozzle are compared at various plate positions relative to the isolated jet. Figure 12(a) displays the effects of the surface as it approaches the jet centerline, and Fig. 12(b) shows the effects of the surface as the plate length is extended relative to the nozzle exit. In Fig. 12(a), the noise intensity deviation from the isolated jet reaches a maximum of $-1.0 \mathrm{~dB}$ at the lowest frequency and $0.1 \mathrm{~dB}$ at the highest frequency when the plate is located at $y_{p} / D=-2$. For all plate locations further than $2 D$ away from the centerline, the noise deviations are within $-0.6 d B$ from the isolated jet case at the lowest frequency and $-0.02 d B$ at the highest frequency. When the plate is located at $y_{p} / D=-1$, the noise reaches a maximum $-6.4 d B$ difference from the isolated jet case. From Fig. 12(b), the noise deviation from the isolated jet reaches a maximum of $-5.7 d B,-6.4 d B$, and $-7.7 \mathrm{~dB}$ at the lowest frequency as the plate extends $x_{p} / D=4,10$, and 20 downstream from the nozzle exit, respectively. The maximum $d B$ difference from the isolated jet for each plate position is slightly decreased in magnitude at lower frequencies when compared to the subsonic jet. There is no increase in noise intensity at high frequencies in contrast to the subsonic case.

Fig. 13 shows comparisons of an on-design $M_{j}=1.5$ cold jet. From Fig. 13(a), the noise intensity deviation from the isolated jet reaches a maximum of $-1.0 \mathrm{~dB}$ at the lowest frequency and $0.1 \mathrm{~dB}$ at the highest frequency when the plate is located at $y_{p} / D=-2$ and $x_{p} / D=10$. For all plate locations further than $2 D$ from the centerline, the noise deviations are within $-0.6 d B$ from the isolated jet case at the lowest frequency and $-0.02 d B$ at the highest frequency. When the plate is located at $y_{p} / D=-1$, the noise reaches a maximum $-6.4 d B$ difference from the isolated jet case at the lowest frequency. In Fig. 13(b), the noise deviation from the isolated jet reaches a maximum of $-6.1 d B,-6.4 d B$, and $-8.1 d B$ as the plate extends to $x_{p} / D=4,10$, and 20 from the nozzle exit, respectively. In regards to the magnitude of the maximum difference and general trend of the spectra, the predictions of the jet and plate cases relative to the isolated jet case are in close agreement with the over-expanded jet. In Fig. 13(b), there is a $0.4 d B$ increase in magnitude of noise intensity deviation with the plate located at $y_{p} / D=-1$ and extending $x_{p} / D=4$ and 20 downstream when compared to the over-expanded jet in Fig. 12(b) at lower frequencies. At the higher frequencies there is no notable difference from the over-expanded jet.

The comparisons described above are representative of the other jet conditions analyzed in this study. As the jet approaches the plate there is a consistently larger difference in the noise spectrum from the isolated jet at lower frequencies. This is due to the surface having a larger effect on the flow further downstream 
from the nozzle exit. It has been shown by Brooks et al. ${ }^{27}$ and Podboy ${ }^{28}$ that peak noise sources are located near the nozzle exit at higher frequencies and lower frequency peak noise sources are located multiple nozzle diameters downstream. The presence of the plate has a larger effect on the jet flow multiple diameters from the nozzle exit and therefore has a larger effect on the aerodynamic source at lower frequencies.

Next, the effect that the plate has on the acoustic source localization is examined by comparing noise source maps of the jet flow for various plate positions using the steady RANS solutions and the integrand of Eqn. 6 near the peak frequency. Contours of SPL per unit St for the cold subsonic $M_{j}=0.513$ jet at a frequency of $1 \mathrm{kHz}(S t \approx 0.3)$ are shown in Fig. 14. In this comparison the plate length extends to $x_{p} / D=10$ and the plate is located at $y_{p} / D=-10,-6,-4,-2$, and -1 from the jet centerline. When the plate is located at $y_{p} / D=-10$ in Fig. 14(a), the noise source distribution is unaffected by the presence of the plate. The two peak noise sources are located $9.5 \mathrm{D}$ downstream from the nozzle exit and are symmetric in magnitude about the jet centerline. There is no significant deformation of the source distribution due to the presence of the plate further than $2 D$ from the jet centerline. As the plate approaches the jet at $y_{p} / D=-2$ shown in Fig. 14(d), the peak magnitude of the source closest to the plate is decreased by $\approx 1 d B$ and the location is unchanged. When the plate is located at $y_{p} / D=-1$, shown in Fig. 14(e), the peak noise source furthest from the plate is decreased in magnitude by $\approx 1 \mathrm{~dB}$ and is shifted $0.5 D$ upstream relative to the $y_{p} / D=-10$ case shown in Fig. 14(a). The peak noise source distribution closest to the plate is significantly deformed. The peak magnitude is decreased by $\approx 6 d B$ and is shifted $1 D$ upstream relative to the $y_{p} / D=-10$ case. An additional strong noise source is also formed close to the plate further downstream at $x / D=14$. This is a distinguishable difference from the other comparisons and could be explained by an observed increase in TKE past the trailing edge of the plate. Figure 14 shows that the magnitude of the peak acoustic source closest to the plate decreases as the plate surface approaches the jet centerline, and the magnitude of the peak acoustic source furthest from the plate is not significantly altered until the plate is located at $y_{p} / D=-1$ from the jet centerline. The location of the peak noise sources are unaltered until the plate is located at $y_{p} / D=-1$. These comparisons represent trends that are consistent for all jet conditions analyzed.

Overall, the results show that the plate has a larger effect on the acoustic source as it approaches the jet centerline and as the plate length extends further downstream. As the effective jet impingement area of the plate is increased, the acoustic intensity radiating from the jet decreases. This result does not account for additional sources produced by the jet interacting with the surface. To quantify the effect of a nearby surface on the jet noise source, the non-dimensional number $\Gamma$ is used, as described in Eqn. 5. The overall sound pressure level (OASPL) is predicted over a frequency range of $20 \mathrm{~Hz}$ to $100 \mathrm{kHz}$ for all plate locations and jet conditions described in Tables 1 and 2 using the steady RANS solutions and Eqn. 6 at $\psi=\pi / 2$. The installed jet predictions of OASPL are subtracted from the isolated jet predictions on a PSD basis. Figure 15 shows the absolute value of $\triangle$ OASPL as a function of $\Gamma$. The physics of the jet flow and airframe interaction dictate the value of $\Gamma$, and the value of $\Gamma$ expresses how large an effect the jet airframe interaction has on the jet noise source. The data collapse shows a critical value of $\Gamma \approx 1$. As the cross-stream length scales $y_{p}\left(y_{p}-D / 2\right)$ decrease to the equivalent of the product of the jet and airframe length scales $\left(D_{j} x_{p} \tan \left[\delta_{\eta}\right]\right), \Gamma$ increases from 0 to 1 . In the region, $0<\Gamma \lesssim 1$, the cross-stream length scales are dominant and the physical quantities of the aerodynamic flow are not sufficiently altered to have a significant effect on the jet noise source. In Fig. 15 , small values of $\Gamma$ approaching $\approx 1$ have $\triangle$ OASPL less than $0.5 d B$ and are considered negligible. As the jet spreading angle, fully expanded jet diameter, or surface length increases the product of the jet and airframe length scales surpasses the cross-stream length scales and $\Gamma$ increases beyond unity. As a result of the dominance of the jet and airframe length scales in this region, $1<\Gamma<\infty$, the physical quantities of the aerodynamic flow are sufficiently altered and have a large effect on the jet noise source. As shown in Fig. 15, values of $\Gamma>1$ result in a range of $\triangle$ OASPL from $2.0 d B$ to $2.75 d B$. It is observed that $\triangle$ OASPL increases as $\Gamma$ increases.

Calculation of $\Gamma$ can be a useful tool when performing propulsion airframe aeroacoustics (PAA) analysis. When analyzing the noise propagation for a jet airframe interaction corresponding to $\Gamma<<1$, the airframe is expected to have a negligible effect on the jet noise source. For instance, a supersonic on-design $M_{j}=1.5$ cold jet with an airframe surface located at $x_{p} / D=20$ and $y_{p} / D=4$ corresponds to $\Gamma=0.099$ and $\Delta$ OASPL $=0.083 d B$ in Fig. 15. Since $\Gamma<<1$ and there is a negligible difference in the free-field jet noise source, the isolated jet aerodynamic source model along with a tailored Green's function can used for a prediction that includes propagation about the airframe. A $M_{j}=0.985$ jet with an airframe surface located at $x_{p} / D=4$ and $y_{p} / D=1$ corresponds to $\Gamma=5.063$ and $\triangle \mathrm{OASPL}=2.47 \mathrm{~dB}$ in Fig. 15 . The large value of $\Gamma$ results 
in a large difference from the free-stream noise prediction; therefore a separate aerodynamic source model is required for further analysis. An isolated jet aerodynamic source model can be used for a PAA analysis with small values of $\Gamma$. For large values of $\Gamma$, some noise reduction can be attributed to the change in the jet noise source and not to shielding effects. Recent studies have investigated the effects of liners on propulsion airframe surfaces. ${ }^{18}$ The effectiveness of airframe liners is associated with the location of the airframe relative to the jet flow. The implications of $\Gamma$ could be useful in future PAA liner studies.

$\Gamma$ can include additional terms involving multiple jet streams or account for the boundary layer thickness on the airframe. An equivalent parameter for the fully expanded jet diameter and jet spreading angle can be developed for a dual-stream jet. However, a new model would be required for the estimation of the jet spreading angle. Inclusion of boundary layer effects will increase the effect of the cross-stream length scales. The airframe boundary layer thickness can be subtracted from the cross-stream length scales. A model to estimate the boundary layer thickness would be required. The general effect of the airframe on the jet noise source as a function of $\Gamma$ is expected to be consistent for these cases but may have a different critical value.

\section{Conclusion}

This study is motivated by low-noise aircraft designs that exhibit situations where the jet exhaust interacts with an airframe surface and to develop an understanding of the effect of nearby surfaces on the aerodynamic noise sources of jet flows. A simplified analysis is performed to examine the variation of the aerodynamics, acoustic source, and far-field acoustic intensity as a large flat plate is moved relative to the nozzle exit. Steady RANS solutions are found using laboratory nozzle and flat plate geometries to study the aerodynamic changes in the field-variables and turbulence statistics. The semi-empirical fine-scale mixing noise model of Tam and Auriault is used to predict the noise produced by the jet and to examine the integrand of the acoustic source. The aerodynamic and noise prediction models are validated by comparing results with PIV and free-field acoustic data, respectively. The variation of the field-variables, turbulence statistics, and noise are examined by comparing predictions from various plate configurations with an isolated jet. The analysis includes multiple jet operating conditions.

Results of the aerodynamic assessment show that nearby solid surfaces change the aerodynamic characteristics of the jet flow-field, even if the jet centerline is multiple diameters away from a solid surface. The effect of the surface is amplified as it approaches the jet centerline and as the plate length extends further downstream. The induced change in the aerodynamic flow-field is shown to have a direct effect on the source of jet noise. The installed noise predictions relative to the isolated jet show that the acoustic intensity originating from the jet aerodynamic source is decreased as the effective impingement surface area of the plate is increased.

A non-dimensional number is developed to quantify the effect of the surface on the jet aerodynamic source based on the jet condition and surface location parameters. The reference number $\Gamma$ physically describes the ratio of the product of the jet and airframe length scales over the cross-stream length scales. The evaluation of $\Gamma$ over the range of surface locations and jet conditions yields a critical value of $\Gamma \approx 1$. Small values of $\Gamma$ suggest that airframe effects on the jet flow are negligible. Large values of $\Gamma$ imply that the effects of the surface on the jet aerodynamic source are very large. This simple calculation of $\Gamma$ can be used as a basic guide to determine if the aerodynamic source is affected by the airframe relative to the equivalent isolated jet aerodynamic source. Additional terms can be included with $\Gamma$ to capture additional physical effects.

\section{Acknowledgments}

The first author is thankful for the support and motivation from his academic advisor, Professor Chris Fuller. The authors are grateful for continuous support from the National Aeronautics and Space Administration (NASA) Fundamental Aeronautics Program (FAP) High Speed Project (HSP). Part of this work is supported by NASA through the National Institute of Aerospace (NIA) cooperative agreement number NNL09AA00A. Drs. James Bridges and Mark P. Wernet of the NASA Glenn Research Center are gratefully acknowledged for use of their experimental data. 


\section{References}

${ }^{1}$ Papamoschou, D. and Mayoral, S., "Experiments on Shielding of Jet Noise by Airframe Surfaces," 15th AIAA/CEAS Aeroacoustics Conference, Miami, Florida, 11 - 13 May, AIAA Paper 2009-3326, 2009.

${ }^{2}$ Huang, C. and Papamoschou, D., "Numerical Study of Noise Shielding by Airframe Structures," 14th AIAA/CEAS Aeroacoustics Conference, Vancouver, British Columbia Canada, 5 - 7 May, AIAA Paper 2008-2999, 2008.

${ }^{3}$ Welge, H. R., Nelson, C., and Bonet, J., "Supersonic Vehicle Systems for the 2020 to 2035 Timeframe," 28th AIAA Applied Aerodynamics Conference, Chicago, Illinois, June 28 - July 1, AIAA Paper 2010-4930, 2010.

${ }^{4}$ Welge, H. R., Bonet, J., Magee, T., Chen, D., Hollowell, S., Kutzmann, A., Mortlock, A., Stengle, J., Nelson, C., Adamson, E., Baughcum, S., Britt, R. T., Miller, G., and Tai, J., "N+2 Supersonic Concept Development and Systems Integration," NASA/CR 2010-216842, 2010.

${ }^{5}$ Morgenstern, J. M., Stelmach, M., and Jha, P. D., "Advanced Concept Studies for Supersonic Commercial Transports Entering Service in 2030-35 (N+3)," 28th AIAA Applied Aerodynamics Conference, Chicago, Illinois, June 28 - July 1, AIAA Paper 2010-5144, 2010.

${ }^{6}$ Sokhey, J. S. and Kube-McDowell, M., "Low Noise Highly Variable Cycle Nozzle for Next Generation Supersonic Aircraft," NASA ARMD Fundamental Aeronautics Program Technical Conference, 2008.

${ }^{7}$ Thomas, R. H., Burley, C. L., and Olson, E. D., "Hybrid Wing Body Aircraft System Noise Assessment with Propulsion Airframe Aeroacoustic Experiments," 16th AIAA/CEAS Aeroacoustics Conference, Stockholm, Sweden, 7 - 9 June, AIAA Paper 2010-3913, 2010.

${ }^{8}$ Czech, M. J., Thomas, R. H., and Elkoby, R., "Propulsion Airframe Aeroacoustic Integration Effects for a Hybrid Wing Body Aircraft Configuration," 16th AIAA/CEAS Aeroacoustics Conference, Stockholm, Sweden, 7 - 9 June, AIAA Paper 2010-3912, 2010.

${ }^{9}$ Tinetti, A. F., Dunn, M. H., and Pope, S., "Fast Scattering Code (FSC) User's Manual, Version 2.0," NASA CR 2006-214510, Oct. 2006.

${ }^{10}$ Agarwal, A., Dowling, A. P., and Graham, W., "A Ray Tracing Approach to Calulate Acoustic Shielding by the Silent Aircraft Airframe," 12th AIAA/CEAS Aeroacoustics Conference, Cambridge, Massachussetts, 8 - 10 May, AIAA Paper 2006$2618,2006$.

${ }^{11}$ Donaldson, C. D. and Snedeker, R. S., "A Study of Free Jet Impingement. Part 1. Mean Properties of Free and Impinging Jets," Journal of Fluid Mechanics, Vol. 45, 1971, pp. 281-319.

${ }^{12}$ Lamont, P. J. and Hunt, B. L., "The Impingement of Underexpanded, Axisymmetric Jets on Perpendicular and Inclined Flat Plates," Journal of Fluid Mechanics, Vol. 100, 1980, pp. 471-511.

${ }^{13}$ Sawyer, R. A., "Two-dimensional Reattaching Jet Flows Including the Effects of Curvature on Entrainment," Journal of Fluid Mechanics, Vol. 17, 1963, pp. 481-498.

${ }^{14} \mathrm{Al}-\mathrm{Qutub}, \mathrm{A} . \mathrm{M}$. and Budair, M. O., "Experiments on the Flow Over a Flat Surface Impinged by a Supersonic Jet," 31st AIAA/ASME/SAE/ASEE Joint Propulsion Conference and Exhibit, San Diego, California, 10 - 12 July, AIAA Paper 1995-2935, 1995.

${ }^{15}$ Tam, C. K. W. and Auriault, L., "Jet Mixing Noise from Fine-Scale Turbulence," AIAA Journal, Vol. 37, No. 2, 1999, pp. $145-153$.

${ }^{16}$ Bridges, J. and Wernet, M. P., "The NASA Subsonic Jet Particle Image Velocimetry (PIV) Dataset," NASA/TM-2011$216807,2011$.

${ }^{17}$ Bridges, J. and Brown, C. A., "Validation of the Small Hot Jet Acoustic Rig for Aeroacoustic Research," 11th AIAA/CEAS Aeroacoustics Conference, Monterey, California, 23 - 25 May, AIAA Paper 2005-2846, 2005.

${ }^{18}$ Thomas, R. H., Czech, M. J., and Doty, M. J., "High Bypass Ratio Jet Noise Reduction and Installation Effects Including Shielding Effectiveness," 51st AIAA Aerospace Sciences Meeting, Grapevine, Texas, 7 - 10 January, AIAA Paper 2013-0541, 2013.

${ }^{19}$ Lau, J. C., "Effects of Exit Mach Number and Temperature on Mean-Flow and Turbulence Characteristics in Round

Jets," Journal of Fluid Mechanics, Vol. 105, 1981, pp. 193-218.

20 "Fun3D Manual," NASA Langley Research Center, Hampton, VA, Aug. 2012, http://fun3d.larc.nasa.gov/.

${ }^{21}$ Menter, F. R., "Two-Equation Eddy-Viscosity Turbulence Models for Engineering Applications," AIAA Journal, Vol. 32, No. 8, 1994, pp. 1598-1605.

${ }^{22}$ Jones, W. P. and Launder, B. E., "The Prediction of Laminarization with a Two-Equation Model of Turbulence," International Journal of Heat and Mass Transfer, Vol. 15, 1972, pp. 301-314.

${ }^{23}$ Wilcox, D. C. and Traci, R. M., "A Complete Model of Turbulence," 9th AIAA Fluid and Plasma Dynamics Conference, San Diego, California, 14 - 16 July, AIAA Paper 1976-351, 1976.

${ }^{24}$ Wilcox, D. C., "A Half Century Historical Review of the k- $\omega$ Model," 29th AIAA Aerospace Sciences Meeting, Reno, Nevada, 7 - 10 January, AIAA Paper 1991-0615, 1991.

${ }^{25}$ Georgiadis, N., Yoder, D. A., and Engblom, W. A., "Evaluation of Modified Two-Equation Turbulence Models for Jet Flow Predictions," 44th AIAA Aerospace Sciences Meeting and Exhibit, AIAA 2006-490, 2006.

${ }^{26}$ Morris, P. J. and Farassat, F., "Acoustic Analogy and Alternative Theories for Jet Noise Prediction," AIAA Journal, Vol. 40, No. 4, 2002, pp. 671-680.

${ }^{27}$ Brooks, T. F., Humphreys, W. M., and Plassman, G. E., "DAMAS Processing for a Phased Array Study in the NASA Langley Jet Noise Laboratory," 16th AIAA/CEAS Aeroacoustics Conference, Stockholm, Sweden, 7 - 9 June, AIAA Paper 2010-3780, 2010.

${ }^{28}$ Podboy, G. G., "Jet-Surface Interaction Test: Phased Array Noise Source Localization Results," Proceeding of the ASME Turbo Expo, Copenhagen, Denmark, June 14-18, GT2012-69801, 2012. 


\section{Tables}

Table 1. Plate Locations

\begin{tabular}{|c|c|}
\hline \hline Axial Location of Trailing Edge $\left(x_{p} / D\right)$ & Radial Location Relative to Jet Centerline $\left(y_{p} / D\right)$ \\
\hline 4 & $1,2,4,6,8,10$ \\
\hline 10 & $1,2,4,6,8,10$ \\
\hline 20 & $1,2,4,6,8,10$ \\
\hline
\end{tabular}

Table 2. Jet Operating Conditions

\begin{tabular}{|c|c|c|c|c|c|}
\hline \hline Nozzle & Setpoint & NPR & TTR & $M_{a}$ & $M_{j}$ \\
\hline SMC000 & 3 & 1.197 & 1.000 & 0.5 & 0.513 \\
\hline SMC000 & 7 & 1.861 & 1.000 & 0.9 & 0.985 \\
\hline SMC000 & 27 & 1.357 & 1.814 & 0.9 & 0.678 \\
\hline SMC016 & 11606 & 2.748 & 0.761 & 1.128 & 1.29 \\
\hline SMC016 & 11610 & 3.670 & 0.706 & 1.31 & 1.5 \\
\hline
\end{tabular}

\section{Figures}

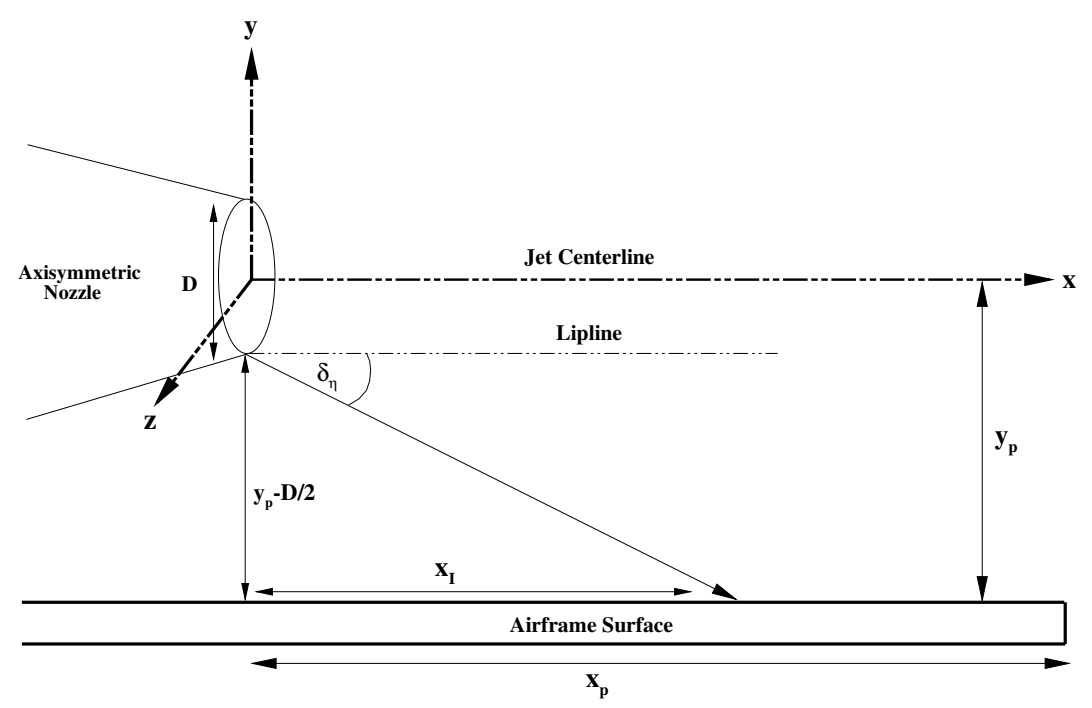

Figure 1. The coordinate system and distance parameters used throughout this study. 


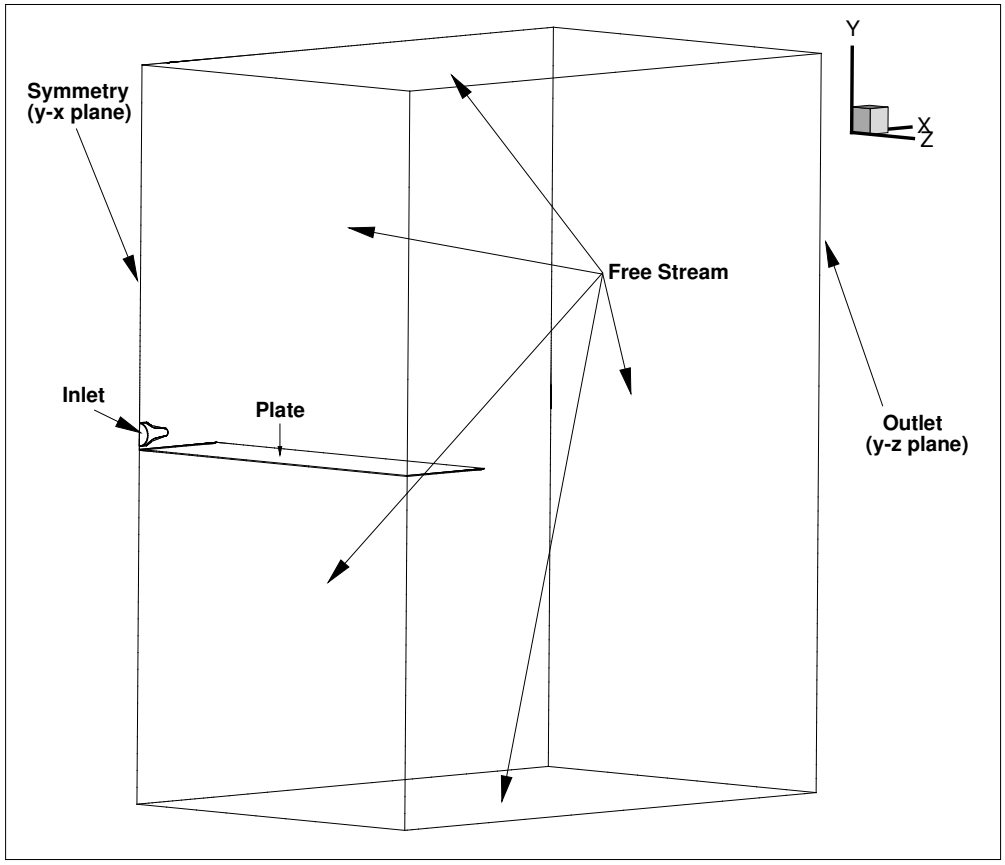

Figure 2. An outline of the computational domain used for the CFD calculations. The edges of the computational domain are shown as black lines. The flow of the jet is in the positive $x$ direction and the coordinate system is centered at the nozzle exit. The domain extends 75 nozzle diameters downstream, 100 diameters in the $y$ direction, and 50 diameters in the $z$ direction. The types of boundaries are labeled.

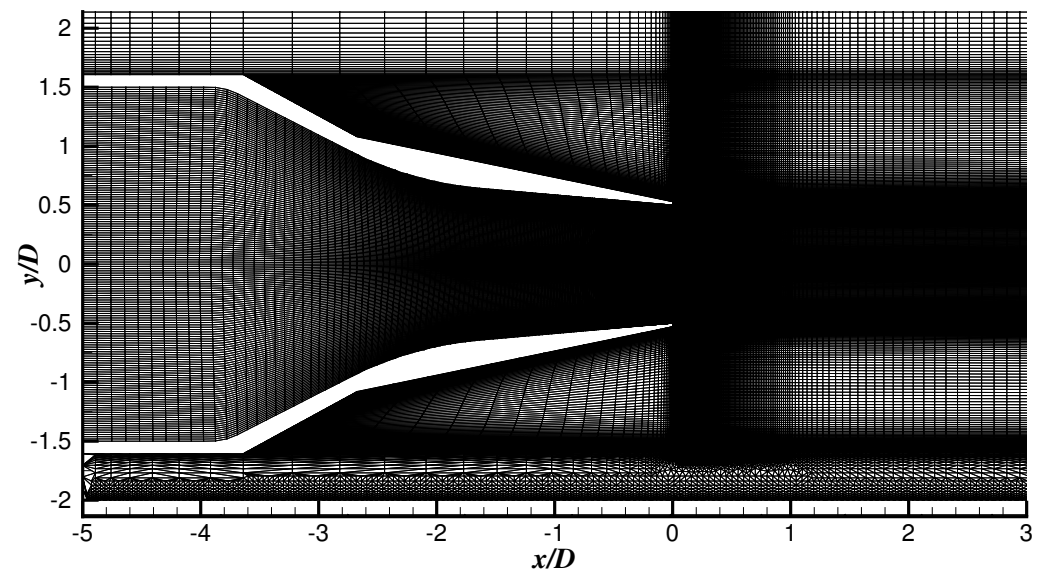

Figure 3. A portion of the computational domain on the $x-y$ plane at $z=0$ showing the structured/unstructured grid of the convergent nozzle and flat plate located $2 D$ away from the centerline. The nozzle geometry matches the NASA Glenn Research Center SMC000 nozzle with an exit diameter of $D=0.0508$ m. The coordinates are normalized by the nozzle exit diameter. 


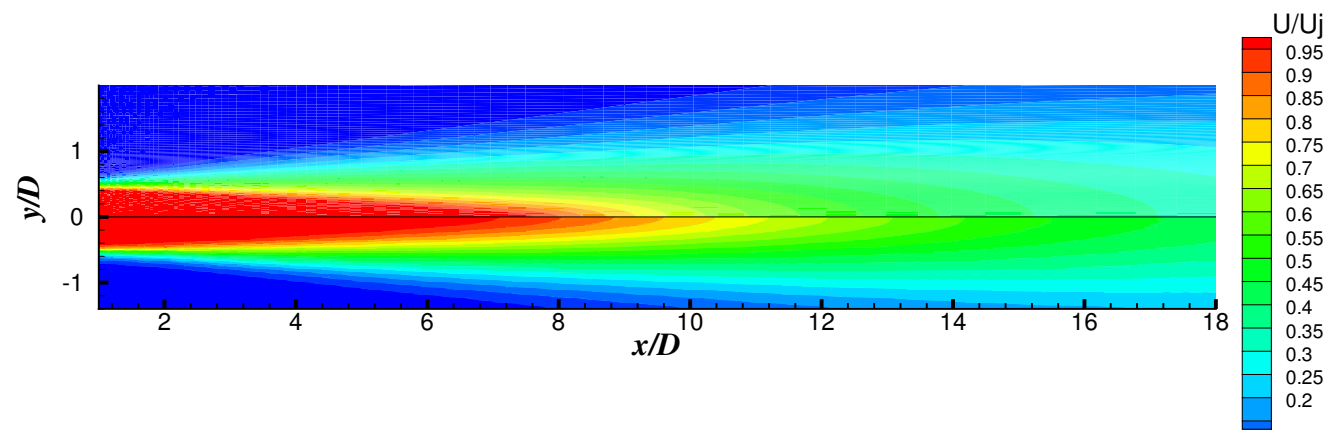

(a) Streamwise Component of Velocity

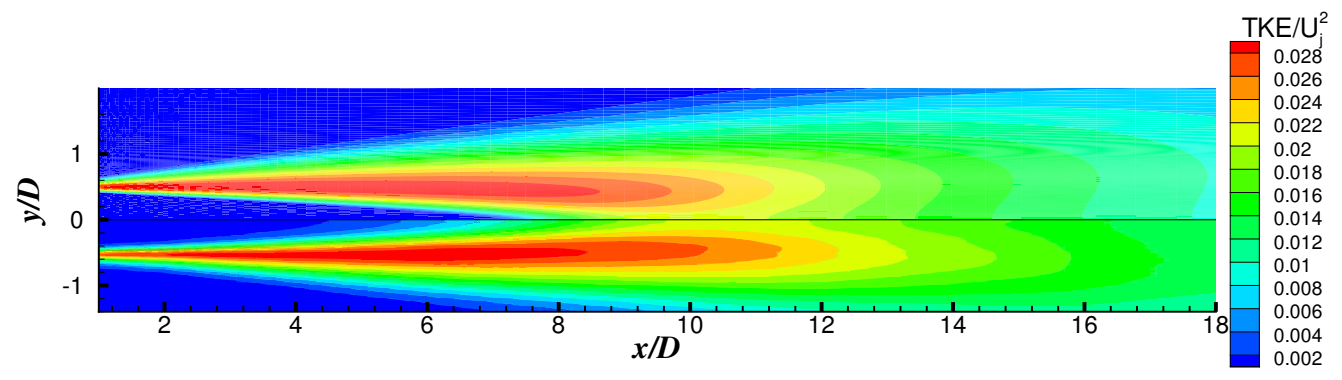

(b) Turbulent Kinetic Energy

Figure 4. Contours of (a) the streamwise velocity component and (b) TKE of the steady RANS solution and PIV data from the experiment of Bridges and Wernet. ${ }^{16}$ The steady RANS solutions are shown on the top half planes and PIV data are shown on the bottom half planes. The streamwise velocity component and TKE are normalized by the fully expanded velocity and the square of the fully expanded velocity respectively. The coordinates are normalized by the nozzle exit diameter $D=0.0508 \mathrm{~m}$. The jet operates at $M_{j}=0.513$ and $T T R=1.00$ from the convergent SMC000 nozzle. 


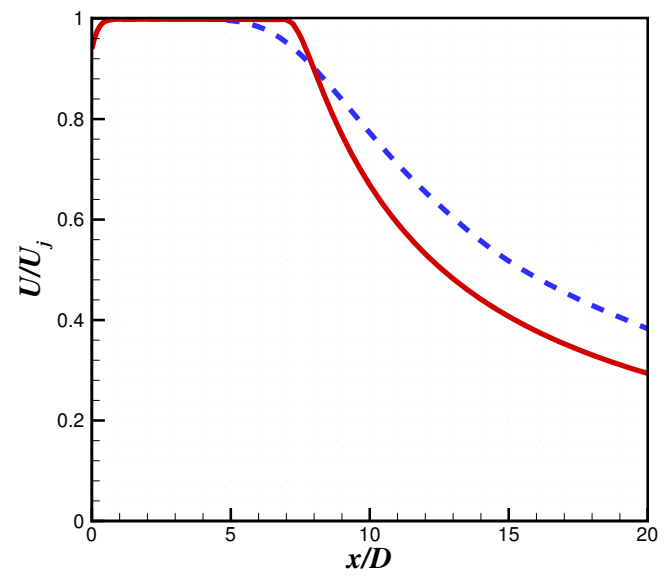

(a) Velocity

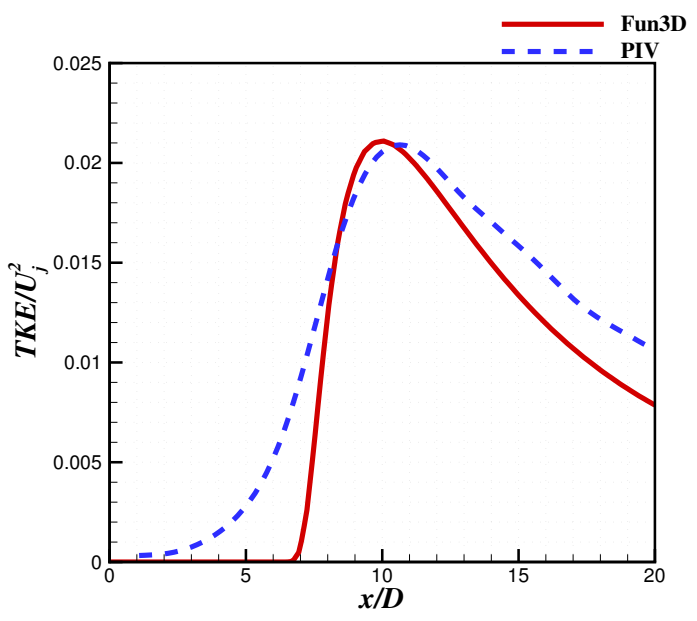

(b) $T K E$

Figure 5. The (a) steady RANS streamwise velocity component and (b) TKE along the jet centerline $(y / D=0$ $\& z / D=0$ ) compared with PIV data from the experiment of Bridges and Wernet. ${ }^{16}$ The streamwise velocity component and TKE are normalized by the fully expanded velocity and the square of the fully expanded velocity respectively. The spatial coordinate is normalized by the nozzle exit diameter $D=0.0508 \mathrm{~m}$. The jet operates at $M_{j}=0.513$ and $T T R=1.00$ from the convergent SMC000 nozzle.

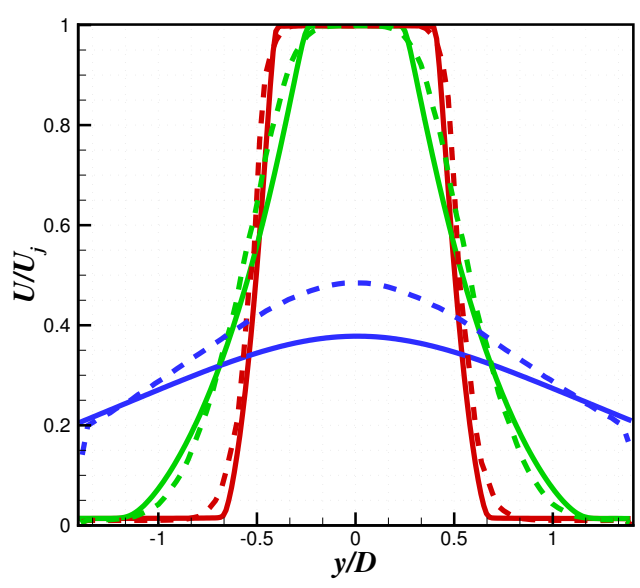

(a) Velocity

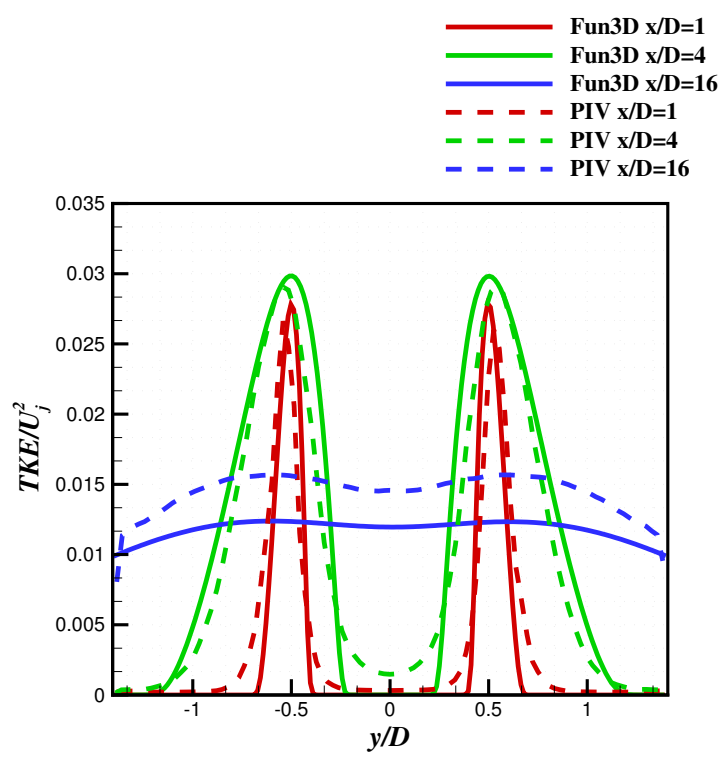

(b) $T K E$

Figure 6. The (a) steady RANS streamwise velocity component and (b) TKE at $x / D=1, x / D=4$, and $x / D=16$ compared with PIV data from the experiment of Bridges and Wernet. ${ }^{16}$ The velocity and TKE are normalized by the fully expanded velocity and the square of the fully expanded velocity respectively. The spatial coordinate is normalized by the nozzle exit diameter $D=0.0508 \mathbf{m}$. The jet operates at $M_{j}=0.513$ and $T T R=1.00$ from the convergent SMC000 nozzle. 


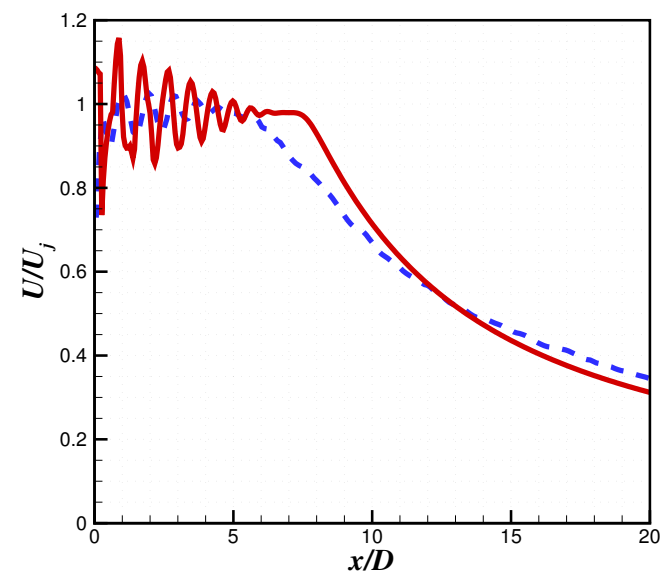

(a) Velocity

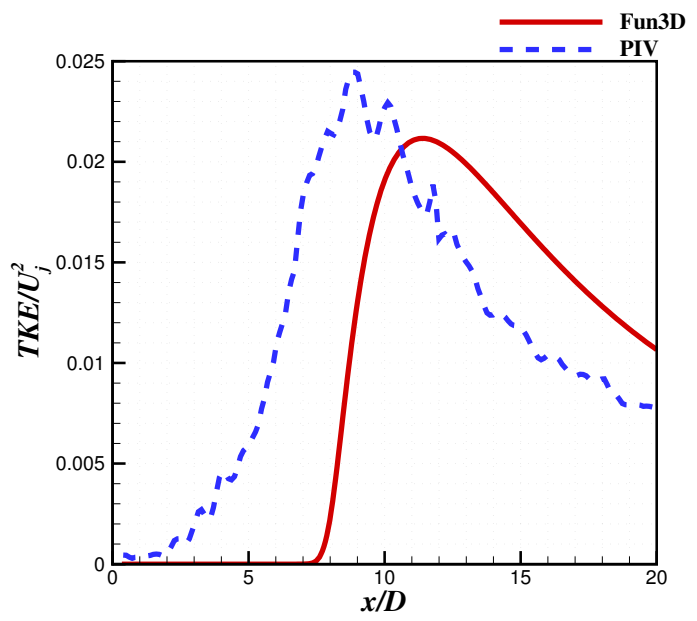

(b) $T K E$

Figure 7. The (a) steady RANS streamwise velocity component and (b) TKE along the jet centerline $(y / D=0$ $\& z / D=0)$ compared with PIV data from the experiment of Bridges and Wernet. ${ }^{16}$ The streamwise velocity component and TKE are normalized by the fully expanded velocity and the square of the fully expanded velocity respectively. The spatial coordinate is normalized by the nozzle exit diameter $D=0.0508 \mathrm{~m}$. The jet operates at $M_{j}=1.29$ and $T T R=1.00$ from the convergent-divergent SMC016 nozzle.

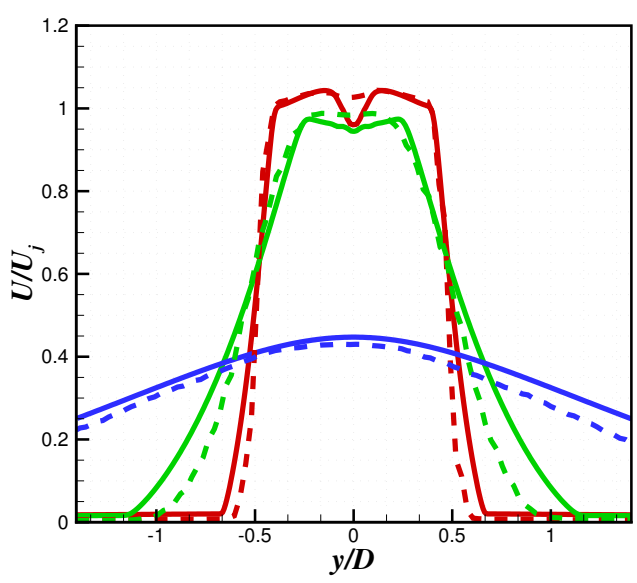

(a) Velocity

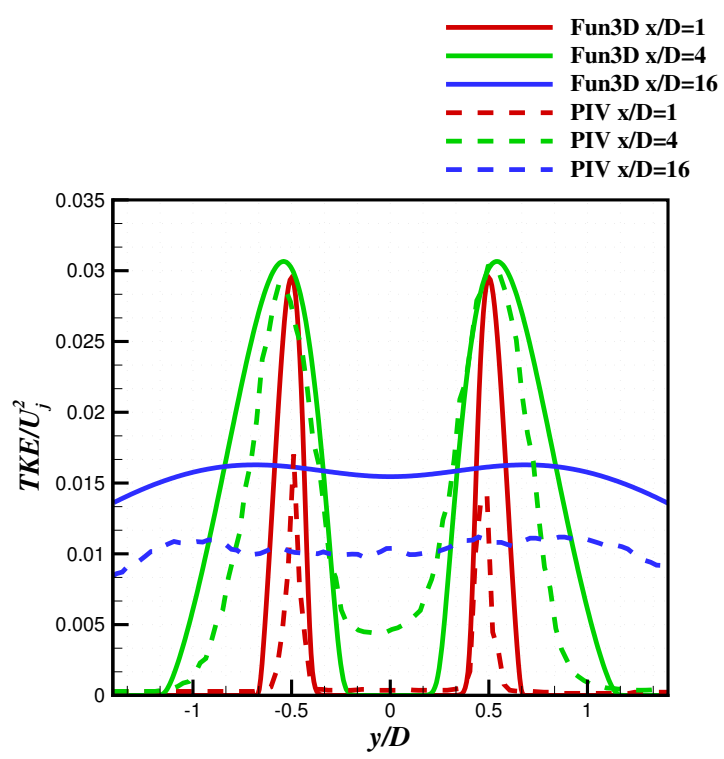

(b) $T K E$

Figure 8. The (a) steady RANS streamwise velocity component and (b) TKE at $x / D=1, x / D=4$, and $x / D=16$ compared with PIV data from the experiment of Bridges and Wernet. ${ }^{16}$ The velocity and TKE are normalized by the fully expanded velocity and the square of the fully expanded velocity respectively. The spatial coordinate is normalized by the nozzle exit diameter $D=0.0508 \mathrm{~m}$. The jet operates at $M_{j}=1.29$ and $T T R=1.00$ from the convergent-divergent SMC016 nozzle. 


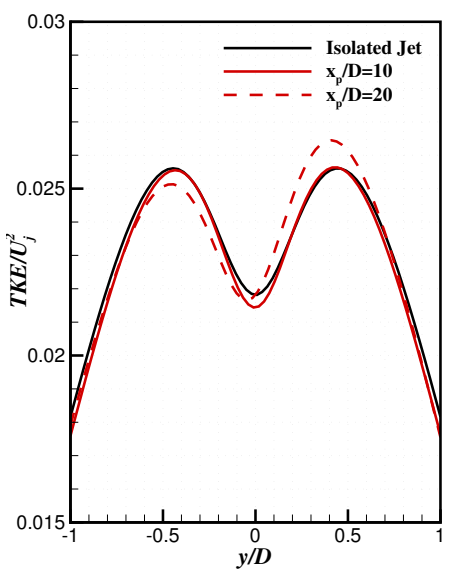

(a) $y_{p} / D=-10$

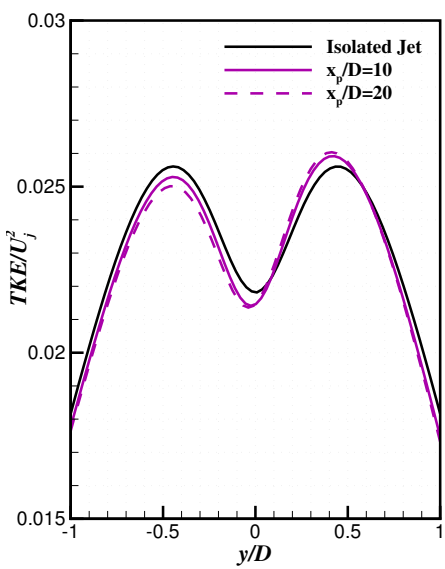

(d) $y_{p} / D=-4$

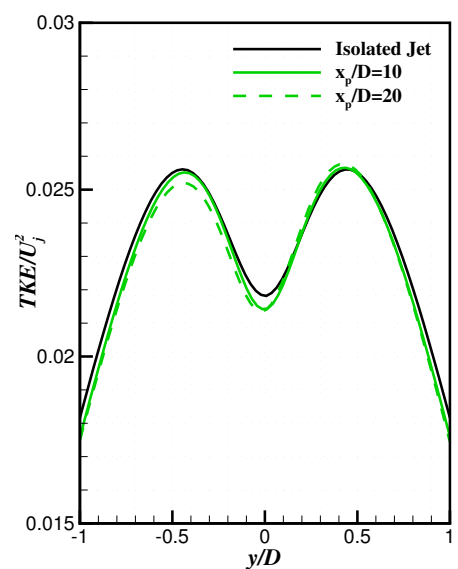

(b) $y_{p} / D=-8$

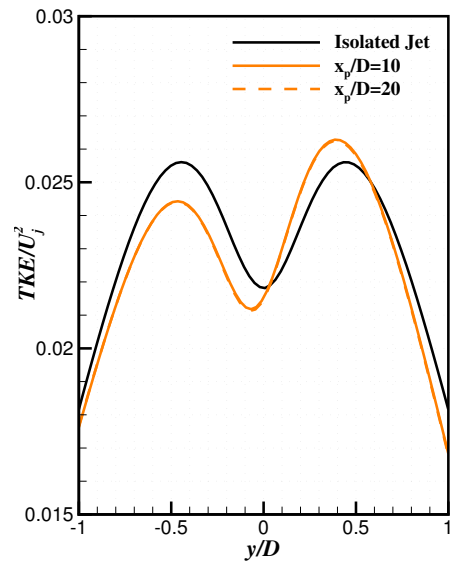

(e) $y_{p} / D=-2$

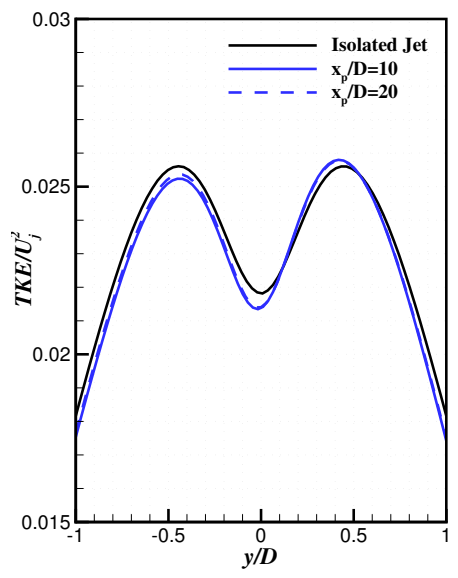

(c) $y_{p} / D=-6$

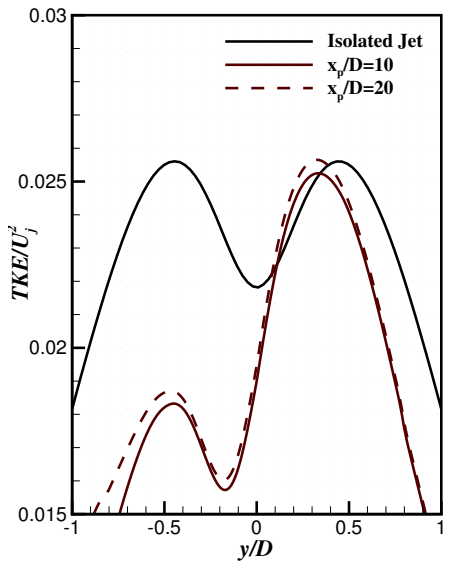

(f) $y_{p} / D=-1$

Figure 9. Radial variation of TKE at $x / D=10$ downstream of the nozzle exit. The spatial coordinate is normalized by the nozzle exit diameter. Parts (a)-(f) show the steady RANS solution with the plate located at $10,8,6,4,2$, and $1 D$ from the jet centerline respectively and extending 10 and $20 D$ downstream from the nozzle $(D=0.0508 \mathrm{~m})$. The jet operates at $M_{j}=0.513$ and $T T R=1.00$ from the convergent SMC000 nozzle. 


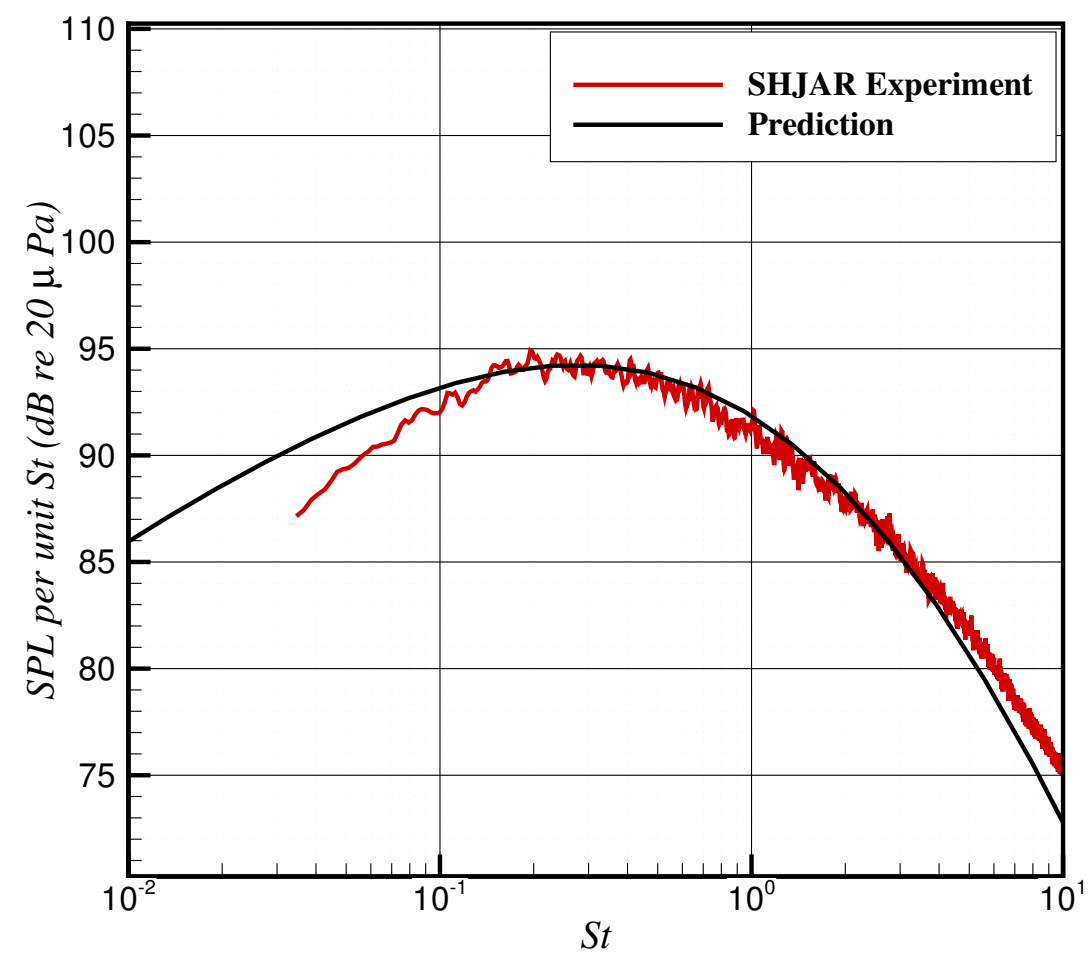

Figure 10. The free-field prediction at $R / D=100$ and $\psi=90$ degrees using the model of Tam and Auriault ${ }^{15}$ compared with the experiment of Bridges and Brown. ${ }^{17}$ The jet operates at $M_{j}=1.00$ and $T T R=1.00$ from the convergent SMC000 nozzle with $D=0.0508 \mathrm{~m}$. 


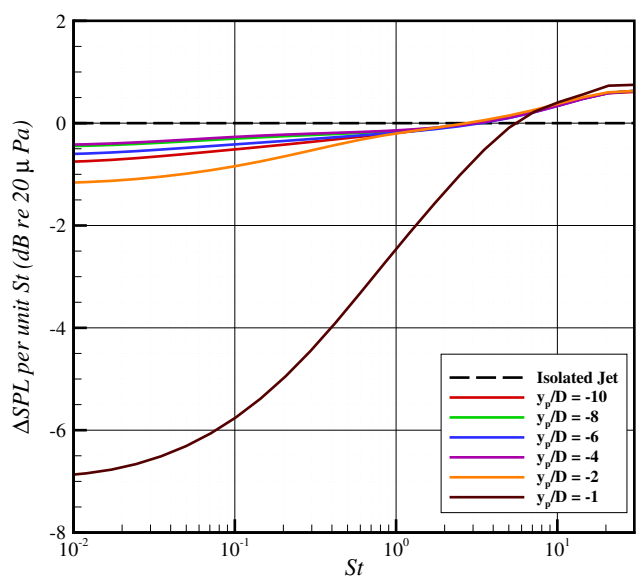

(a) $x_{p} / D=10$

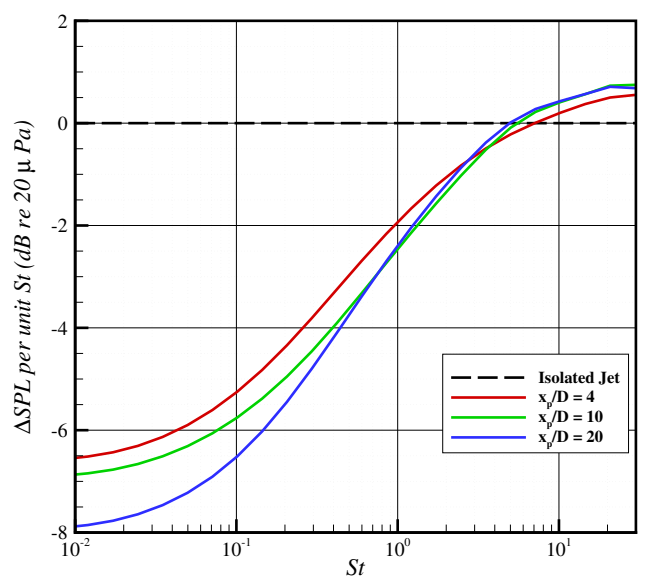

(b) $y_{p} / D=-1$

Figure 11. Attenuation plot of the free-field prediction at $R / D=100$ and $\psi=90$ degrees using the model of Tam and Auriault ${ }^{15}$ for the isolated jet and plate cases. For part (a), the plate is located at 1, 2, 4, 6, 8, and $10 D$ laterally from the jet centerline and extends $10 D$ downstream from the nozzle. For part (b) the plate is located at $1 D$ laterally from the jet centerline and extends 4,10 , and $20 D$ downstream from the nozzle. The jet operates at $M_{j}=0.513$ and $T T R=1.00$ from the convergent SMC000 nozzle with $D=0.0508 \mathrm{~m}$.

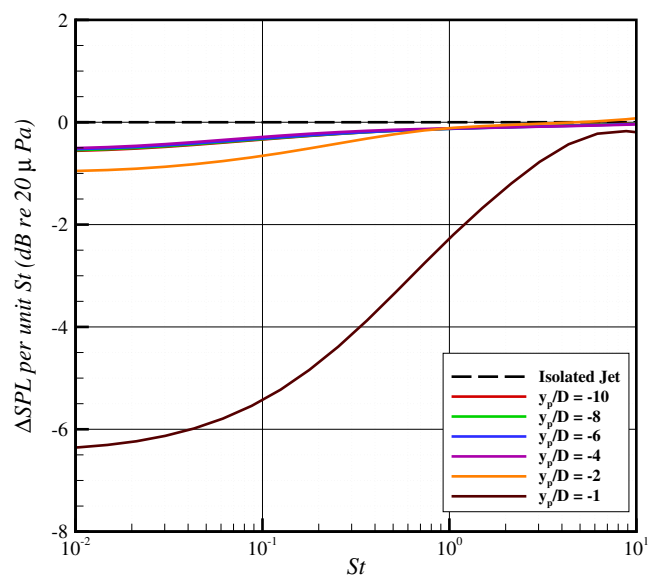

(a) $x_{p} / D=10$

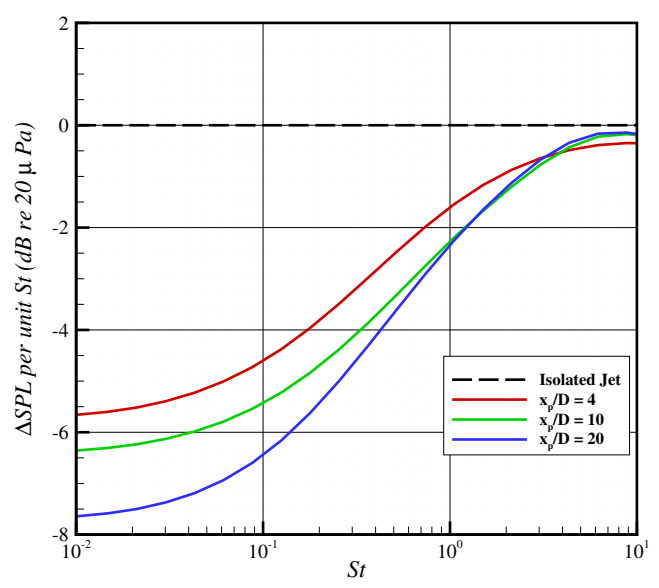

(b) $y_{p} / D=-1$

Figure 12. Attenuation plot of the free-field prediction at $R / D=100$ and $\psi=90$ degrees using the model of Tam and Auriault ${ }^{15}$ for the isolated jet and plate cases. For part (a), the plate is located at 1, 2, 4, 6, 8, and $10 D$ laterally from the jet centerline and extends $10 D$ downstream from the nozzle. For part (b) the plate is located at $1 D$ laterally from the jet centerline and extends 4,10 , and $20 D$ downstream from the nozzle. The jet operates at $M_{j}=1.29$ and $T T R=1.00$ from the convergent SMC016 nozzle with $D=0.0508 \mathrm{~m}$. 


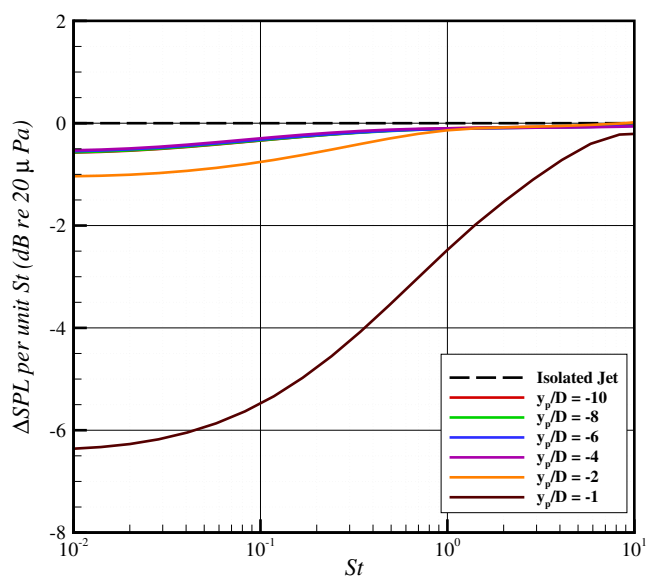

(a) $x_{p} / D=10$

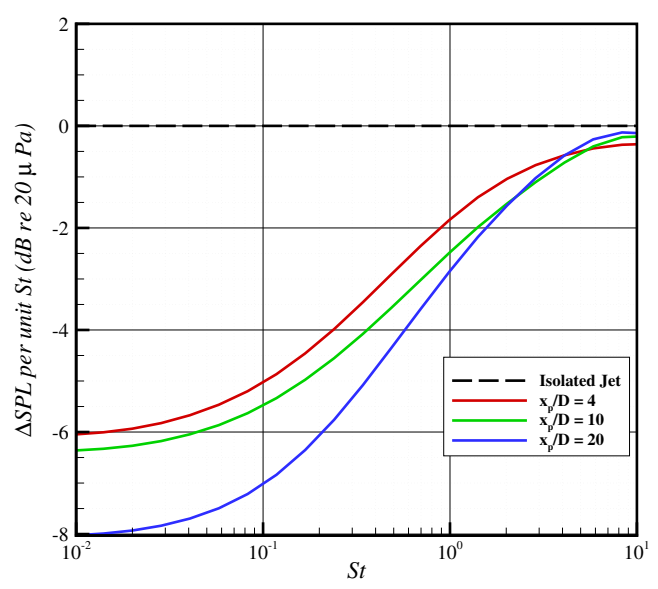

(b) $y_{p} / D=-1$

Figure 13. Attenuation plot of the free-field prediction at $R / D=100$ and $\psi=90$ degrees using the model of Tam and Auriault ${ }^{15}$ for the isolated jet and plate cases. For part (a), the plate is located at 1, 2, 4, 6, 8, and $10 D$ laterally from the jet centerline and extends $10 D$ downstream from the nozzle. For part (b) the plate is located at $1 D$ laterally from the jet centerline and extends 4,10 , and $20 D$ downstream from the nozzle. The jet operates at $M_{j}=1.50$ and $T T R=1.00$ from the convergent SMC016 nozzle with $D=0.0508$ m. 


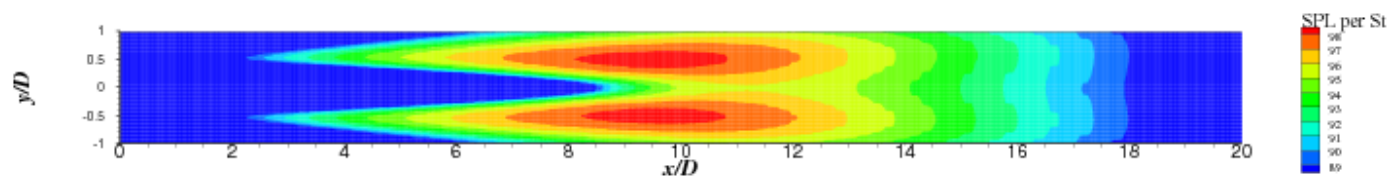

(a) $y_{p} / D=-10$

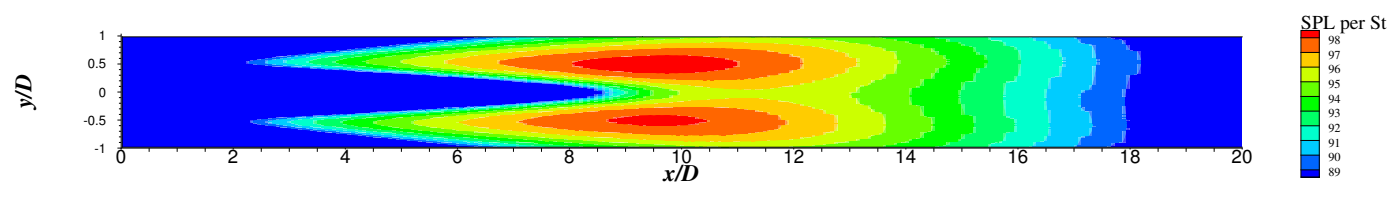

(b) $y_{p} / D=-6$

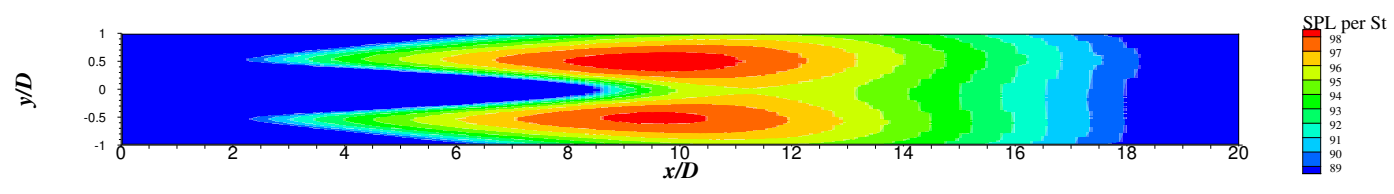

(c) $y_{p} / D=-4$

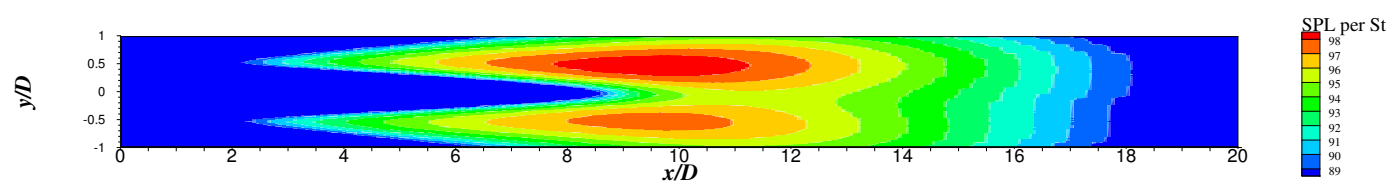

(d) $y_{p} / D=-2$

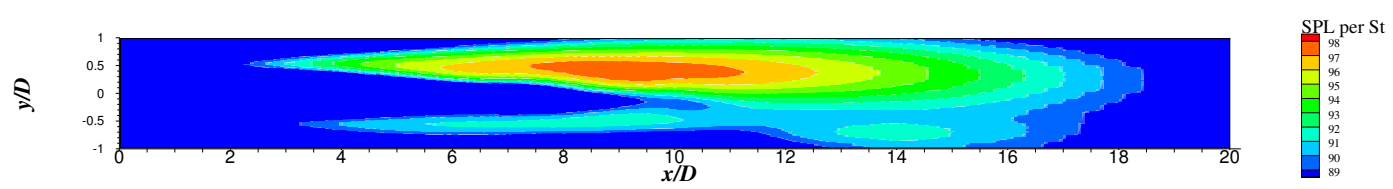

(e) $y_{p} / D=-1$

Figure 14. Contours of peak acoustic source downstream from the nozzle exit at $f=1000 H z(S t \approx 0.3)$. The spatial coordinates are normalized by the nozzle exit diameter. Parts (a)-(e) show the acoustic source with the plate located at $y_{p} / D=-10,-6,-4,-2$, and -1 respectively and extending $10 D$ downstream from the nozzle. The jet operates at $M_{j}=0.513$ and $T T R=1.00$ from the convergent SMC000 nozzle with $D=0.0508 \mathrm{~m}$. 


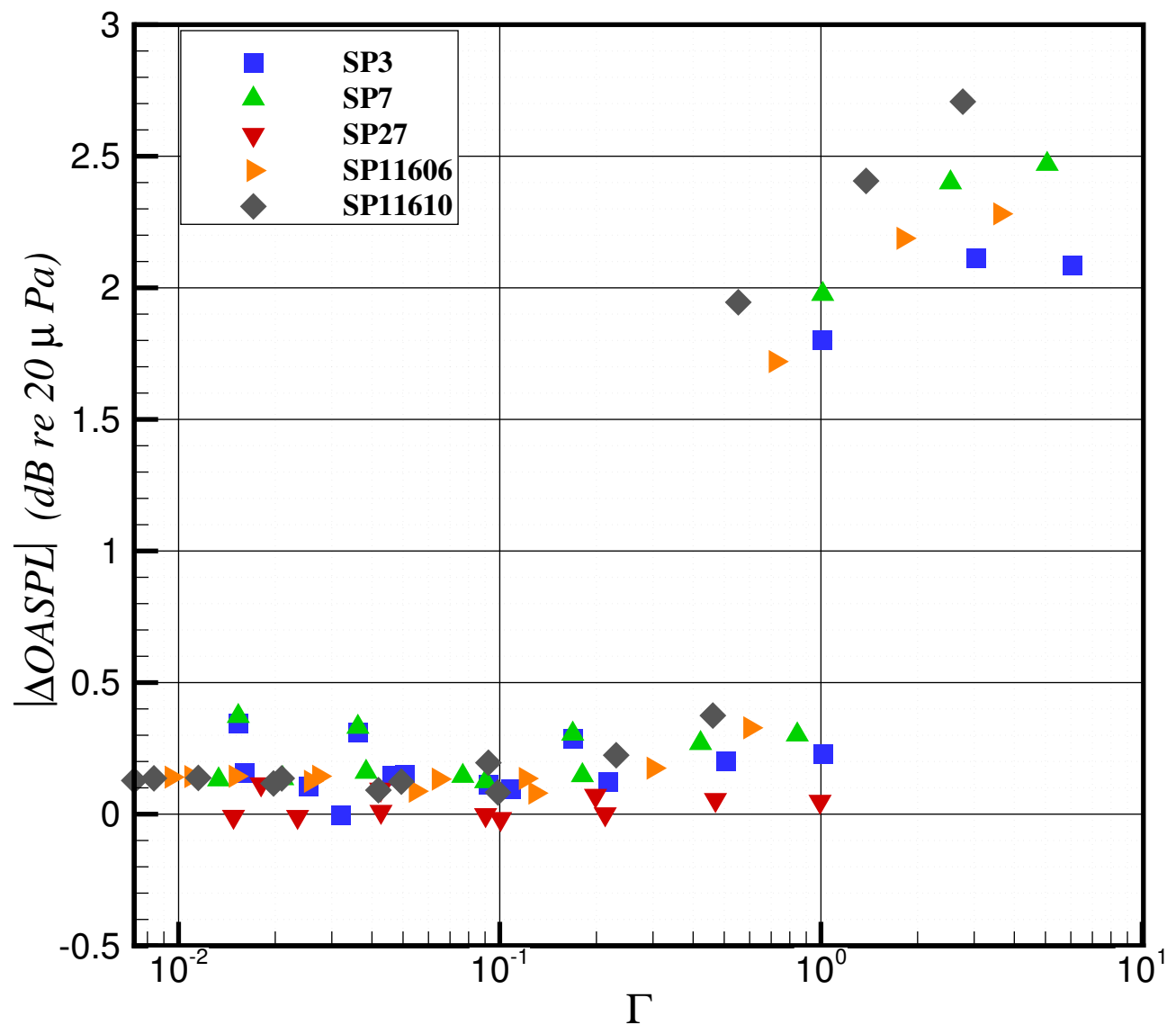

Figure 15. Results of $\triangle O A S P L$ as a function of non-dimensional number $\Gamma$ (Eqn. 5). $\triangle O A S P L$ is calculated relative to the isolated jet case for each airframe configuration and jet condition as listed in Tables 1 and 2 , respectively. 